\title{
One-dimensional gas dynamics equations of a polytropic gas in Lagrangian coordinates: symmetry classification, conservation laws, difference schemes
}

\author{
Vladimir A. Dorodnitsyn ${ }^{a}$, Roman $\operatorname{Kozlov}^{b}$, Sergey V. Meleshko ${ }^{c}$ \\ ${ }^{a}$ Keldysh Institute of Applied Mathematics, Russian Academy of Science, \\ Miusskaya Pl. 4, Moscow, 125047, Russia; \\ e-mail: Dorodnitsyn@Keldysh.com \\ ${ }^{b}$ Department of Business and Management Science, Norwegian School of \\ Economics, Helleveien 30, 5045, Bergen, Norway; \\ e-mail: Roman.Kozlov@nhh.no \\ ${ }^{c}$ School of Mathematics, Institute of Science, Suranaree University of \\ Technology, 30000, Thailand; \\ e-mail: sergey@math.sut.ac.th
}

\begin{abstract}
Lie point symmetries of the one-dimensional gas dynamics equations of a polytropic gas in Lagrangian coordinates are considered. Complete Lie group classification of these equations reduced to a scalar second-order PDE is performed. The classification parameter is the entropy. Noether's theorem is applied for constructing conservation laws. The conservation laws can be represented in the gas dynamics variables. For the basic adiabatic case invariant and conservative difference schemes are discussed.
\end{abstract}

Keyword: Lagrangian gas dynamics, Lie point symmetries, Lie group classification, conservation law, Noether's theorem, numerical scheme.

\section{Introduction}

There is an extensive literature on the Lagrangian gas dynamics (see, for example, 8, 35, 36, and references therein). These studies can be roughly separated on analysis of the gas dynamics equations in the mass Lagrangian coordinates [28, 8] and analysis of second-order PDEs to which these gas dynamics equations can be reduced [36, 35, 32, 34]. These second-order PDEs have variational structure, i.e. they are Euler-Lagrange equations for some Lagrangian function. They are knows as the Lagrangian gas dynamics [8]. In the present paper these Euler-Lagrange equations, which represent gas dynamics equations in mass Lagrangian coordinates, are studied.

Symmetries have always attracted the attention of scientists. One of the tools for studying symmetries is the group analysis method [24, 23, 20, 16, 6], which is a basic method for constructing exact invariant solutions of partial differential equations. The group properties of the gas dynamics equations in Eulerian coordinates were studied in [24, 25]. Extensive group analysis of the 
one-dimensional gas dynamics equations in mass Lagrangian coordinates was given in [1, 2, 15]. Here it should be also mentioned the results of [33], where nonlocal conservation laws of the one-dimensional gas dynamics equations in mass Lagrangian coordinates were found. The authors of [36, 35] analyzed the Euler-Lagrange equations corresponding to the one-dimensional gas dynamics equations in mass Lagrangian coordinates: extensions of the known conservation laws were derived. These conservation laws correspond to special forms of the entropy. Group nature of these conservation laws is given in the present paper. Complete group analysis of the Euler-Lagrange equations of the onedimensional gas dynamics equations of isentropic flows of a polytropic ga: 1 with $\gamma=2$ was given in 32 .

As mentioned above, besides assisting with the construction of exact solutions, the knowledge of an admitted Lie group allows one to derive conservation laws. Conservation laws provide information on the basic properties of solutions of differential equations. They are also needed in the analyses of stability and global behavior of solutions. Noether's theorem [22] is the tool which relates symmetries and conservation laws. However, an application of Noether's theorem depends on the following condition: the differential equations under consideration need to be presented as Euler-Lagrange equations with appropriate Lagrangian. Among approaches which try to overcome this limitation one can mention here the approaches developed in [31, 17, 5, 30] 2 .

Lie group analysis of difference equations is a very active field of research [19, 37, 14]. In our approach, which we are following in this paper, we discretize differential equations while preserving their Lie point symmetries. Thus differential equations and its Lie group symmetries are a priory given but not a difference model. One then looks for a difference scheme, i.e. difference equations and a mesh, that have the same symmetry group and the same Lie algebra. The basic steps in this direction were done [9, 10, 4, 11, 12, 13, which were summarized in the book [14]. The main idea is that the invariant difference equations and meshes can be constructed with the help of the entire set of difference invariants of the corresponding Lie group.

The present paper is focused on the one-dimensional gas dynamics equations of a polytropic gas in Lagrange coordinates. The objective of the paper is to make Lie group classification of the variational second-order PDE to which the gas dynamics equations can be reduced. The classification is carried out with respect to the entropy function. Then, we use variational and divergent symmetries to construct conservation laws by applying Noether's theorem. In the second part of the paper we consider discrete schemes which preserve symmetries and conservation laws for the basic adiabatic case of the polytropic gas.

The paper is organized as follows: Section 2 introduces equations of gas dynamics, their variational formulation and Lie point symmetries. In section 3 we consider the general case and the corresponding conservation laws. Then, we

\footnotetext{
${ }^{1}$ The hyperbolic shallow water equations are equivalent to the gas dynamics equations for a polytropic gas with $\gamma=2$.

${ }^{2}$ Therein one can find more details and references.
} 
present the cases with additional symmetries and conservation laws in section 4. Discrete schemes for the general case are discussed in section 5. Finally, section 6 gives concluding remarks.

\section{Gas dynamics equations}

In this section we present the one-dimensional gas dynamics equations for a polytropic gas. We start with Euler coordinates and show how to change to Lagrangian ones. It is shown how one can reduce the gas dynamics equations to a scalar second-order PDE, which has variational structure.

\subsection{The gas dynamics equations in Eulerian coordinates}

The gas dynamics equations of a polytropic gas are

$$
\begin{gathered}
\rho_{t}+u \rho_{x}+\rho u_{x}=0, \\
\rho\left(u_{t}+u u_{x}\right)+p_{x}=0, \\
S_{t}+u S_{x}=0,
\end{gathered}
$$

where $\rho$ is the density, $u$ is the velocity and

$$
p=S \rho^{\gamma}, \quad \gamma \neq 0,1,
$$

is the pressure. Here $S$ is the function depending on the entropy $\tilde{S}$ of the gas. For a polytropic gas this relation takes the form [26]

$$
S=R e^{\left(\tilde{S}-\tilde{S}_{0}\right) / c_{v}},
$$

where $R$ is the gas constant, $c_{v}$ is the dimensionless specific heat capacity at constant volume and $\tilde{S}_{0}$ is constant.

Note that due to (2.2) the last equation (2.1c) is equivalent to

$$
p_{t}+u p_{x}+\gamma p u_{x}=0
$$

\subsection{Eulerian and Lagrangian coordinates}

For one-dimensional gas dynamics one can introduce mass Lagrangian coordinate $s$ in Eulerian coordinates $(t, x)$ as

$$
d s=\rho d x-\rho u d t
$$

or

$$
s_{x}=\rho, \quad s_{t}=-\rho u .
$$

We also need the time differentiation in Lagrangian coordinates $(t, s)$, which is called the material derivative,

$$
D_{t}^{L}=D_{t}^{E}+u D_{x}
$$


where $D_{t}^{E}$ and $D_{x}$ are the total differentiations with respect to $t$ and $x$ in Euler coordinates. Thus we arrive at the gas dynamics equations in Lagrangian coordinates:

$$
\begin{gathered}
\left(\frac{1}{\rho}\right)_{t}=u_{s}, \\
u_{t}+p_{s}=0, \\
S_{t}=0 .
\end{gathered}
$$

Note that the equation (2.4) takes the form

$$
p_{t}+\gamma \rho p u_{s}=0
$$

We remark that we use a non-point change of the independent variables.

\subsubsection{Variational formulation}

Let us introduce variable $\varphi$ as the potential for equation (2.8a):

$$
\varphi_{t}=u, \quad \varphi_{s}=\frac{1}{\rho}
$$

and solve equation (2.8c) as

$$
S=S(s) \text {. }
$$

Then, with the help of (2.2) we can rewrite the remaining equation (2.8b) as the following second-order PDE [7]:

$$
\varphi_{s}^{\gamma} \varphi_{t t}-\gamma S \varphi_{s}^{-1} \varphi_{s s}+S_{s}=0
$$

This equation will be called the gas dynamics equation in Lagrangian coordinates. It is the variational equation for the Lagrangian

$$
L=\frac{\varphi_{t}^{2}}{2}-\frac{S(s)}{\gamma-1} \varphi_{s}^{1-\gamma} .
$$

Let us recall that the Euler-Lagrange equation for Lagrangian (2.13) is computed as

$$
\frac{\delta L}{\delta \varphi}=\frac{\partial L}{\partial \varphi}-D_{t}^{L}\left(\frac{\partial L}{\partial \varphi_{t}}\right)-D_{s}\left(\frac{\partial L}{\partial \varphi_{s}}\right)=0
$$

A symmetry

$$
X=\xi^{t}(t, s, \varphi) \frac{\partial}{\partial t}+\xi^{s}(t, s, \varphi) \frac{\partial}{\partial s}+\eta^{\varphi}(t, s, \varphi) \frac{\partial}{\partial \varphi}
$$

of equation (2.12) provides its conservation law if it satisfies the equation

$$
X L+L\left(D_{t}^{L} \xi^{t}+D_{s} \xi^{s}\right)=D_{t}^{L} B_{1}+D_{s} B_{2},
$$

for some functions $B_{i}(t, s, \varphi), i=1,2$. Here we assume that the operator $X$ is prolonged on the derivatives by means of the standard prolongation formulae [24, 23].

The densities $\left(T^{t}, T^{s}\right)$ of the conservation laws are given by the formulae

$$
T^{t}=\xi^{t} L+\left(\eta^{\varphi}-\xi^{t} \varphi_{t}-\xi^{s} \varphi_{s}\right) \frac{\partial L}{\partial \varphi_{t}}, \quad T^{s}=\xi^{s} L+\left(\eta^{\varphi}-\xi^{t} \varphi_{t}-\xi^{s} \varphi_{s}\right) \frac{\partial L}{\partial \varphi_{s}}
$$




\subsubsection{Relation between conservation laws in Lagrangian and Eule- rian coordinates}

The operators of the total differentiation $D_{t}^{L}$ and $D_{s}$ in Lagrangian coordinates $(t, s)$ and the operators of the total differentiation $D_{t}^{E}$ and $D_{x}$ in Eulerian coordinates $(t, x)$ are related as follows

$$
D_{t}^{L}=D_{t}^{E}+\varphi_{t} D_{x}=D_{t}^{E}+u D_{x}, \quad D_{s}=\varphi_{s} D_{x}=\frac{1}{\rho} D_{x} .
$$

Let $\left(T^{t}, T^{s}\right)$ be a conserved vector in Lagrangian coordinates:

$$
D_{t}^{L} T^{t}+D_{s} T^{s}=0
$$

Using (2.10), we obtain

$$
u_{x}=\varphi_{s}^{-1} \varphi_{t s}
$$

and

$$
D_{t}^{L} T^{t}+D_{s} T^{s}=\varphi_{s}\left(D_{t}^{E}\left(\rho T^{t}\right)+D_{x}\left(\rho u T^{t}+T^{s}\right)\right) .
$$

Thus, the corresponding conserved vector $\left({ }^{e} T^{t},{ }^{e} T^{x}\right)$ in Eulerian coordinates has the coordinates

$$
{ }^{e} T^{t}=\rho T^{t}, \quad{ }^{e} T^{x}=\rho u T^{t}+T^{s} .
$$

\subsection{Equivalence transformations}

The group classification of differential equations depends on representations of arbitrary elements [24]. As the function $S(s)$ is an arbitrary element of equation (2.12), the group classification has to be made with respect to it.

As the first step in the symmetry group classification we find equivalence Lie group transformations. The generator of the equivalence transformations is considered in the form [21]

$$
X^{e}=\xi^{t} \frac{\partial}{\partial t}+\xi^{s} \frac{\partial}{\partial s}+\eta^{\varphi} \frac{\partial}{\partial \varphi}+\eta^{S} \frac{\partial}{\partial S}
$$

where all the coefficients of the generator $X^{e}$ depend on $(t, s, \varphi, S)$.

Calculations show that the Lie algebra corresponding to the equivalence Lie group of equation (2.12) consists of the generators

$$
\begin{array}{rlrl}
X_{1}^{e}=\frac{\partial}{\partial \varphi}, \quad X_{2}^{e}=\frac{\partial}{\partial t}, \quad X_{3}^{e}=\frac{\partial}{\partial s}, & X_{4}^{e}=t \frac{\partial}{\partial \varphi} \\
X_{5}^{e}=\varphi \frac{\partial}{\partial \varphi}+(\gamma+1) S \frac{\partial}{\partial S}, & X_{6}^{e}=t \frac{\partial}{\partial t}-2 S \frac{\partial}{\partial S} \\
X_{7}^{e}=s \frac{\partial}{\partial s}+(1-\gamma) S \frac{\partial}{\partial S} .
\end{array}
$$

For $\gamma=3$ there are more equivalence transformations corresponding to the generator

$$
X_{*}^{e}=t^{2} \frac{\partial}{\partial t}+t \varphi \frac{\partial}{\partial \varphi} .
$$




\subsection{Group classification of equation (2.12)}

Group classification of equation (2.12) was given in [3]. For the sake of completeness we give it here. The admitted generator has the form

$$
X=\sum_{i=1}^{8} k_{i} Y_{i}
$$

where

$$
\begin{aligned}
Y_{1}=\varphi \frac{\partial}{\partial \varphi}, \quad Y_{2}=t^{2} \frac{\partial}{\partial t}+t \varphi \frac{\partial}{\partial \varphi}, \quad Y_{3}=t \frac{\partial}{\partial t}, \quad Y_{4}=\frac{\partial}{\partial t}, \\
Y_{5}=t \frac{\partial}{\partial \varphi}, \quad Y_{6}=\frac{\partial}{\partial \varphi}, \quad Y_{7}=s \frac{\partial}{\partial s}, \quad Y_{8}=\frac{\partial}{\partial s} .
\end{aligned}
$$

The coefficients $k_{i}$ satisfy the conditions

$$
\begin{aligned}
\left(k_{7} s+k_{8}\right) S_{s}= & \left((1-\gamma) k_{7}+(\gamma+1) k_{1}-2 k_{3}\right) S, \\
& (\gamma-3) k_{2}=0 .
\end{aligned}
$$

Thus, in the generic case $(\gamma \neq 3)$ the kernel of the algebra of the admitted Lie symmetries can be presented by the following basis

$$
\begin{aligned}
X_{1}=Y_{6}=\frac{\partial}{\partial \varphi}, \quad X_{2} & =Y_{4}=\frac{\partial}{\partial t}, \quad X_{3}=Y_{5}=t \frac{\partial}{\partial \varphi} \\
X_{4} & =2 Y_{1}+(\gamma+1) Y_{3}=(\gamma+1) t \frac{\partial}{\partial t}+2 \varphi \frac{\partial}{\partial \varphi} .
\end{aligned}
$$

For $\gamma=3$ the kernel of the admitted Lie symmetries algebra has one additional generator

$$
X_{*}=Y_{2}=t^{2} \frac{\partial}{\partial t}+t \varphi \frac{\partial}{\partial \varphi} .
$$

According to the classifying equation (2.28a ), there exists constants $\alpha, \beta$ and $q$ such that the entropy function satisfies the equation

$$
(\alpha s+\beta) S_{s}=q S .
$$

Let us consider possible cases:

1. $q=0(\alpha \neq 0$ or $\beta \neq 0)$

I this case equation (2.31) gives

$$
S(s)=A_{0}, \quad A_{0}=\text { const. }
$$

2. $q \neq 0$

(a) If $\alpha \neq 0$, then by virtue of the equivalence transformations equation (2.31) can be reduced to the equation

$$
s S_{s}=q S, \quad q \neq 0 .
$$

The general solution of the latter equation is

$$
S(s)=A_{0} s^{q}, \quad A_{0}=\text { const. }
$$


(b) If $\alpha=0$ and $\beta \neq 0$, equation (2.31) can be reduced to

$$
S_{s}=q S, \quad q \neq 0,
$$

which has the general solution

$$
S=A_{0} e^{q s}, \quad A_{0}=\text { const. }
$$

Thus, one needs to study three cases: (2.32), (2.33) and (2.34). Notice that the first case (2.32) corresponds to an isentropic flow of a polytropic gas. It is well-studied, whereas the other cases were not analyzed in the theory of gas dynamics equations. We remark that by virtue of the equivalence transformations one can assume that $A_{0}=1$.

\section{Conservation laws for arbitrary entropy $S(s)$}

In Lagrangian coordinates the conservation law of mass in given by the first equation of the gas dynamics system (2.8), namely

$$
\left(\frac{1}{\rho}\right)_{t}-u_{s}=0
$$

For variational and divergent symmetries we can using Noether's theorem to derive conservation laws. In this section we exploit the generators from the kernel of admitted Lie symmetry algebra.

\subsection{Case $\gamma \neq 3$}

Let us examine symmetries (2.29) of the generic case $\gamma \neq 3$. For Lagrangian (2.13) we get two variational symmetries

$$
Z_{1}=X_{1}=\frac{\partial}{\partial \varphi}, \quad Z_{2}=X_{2}=\frac{\partial}{\partial t}
$$

and one divergent symmetry

$$
Z_{3}=X_{3}=t \frac{\partial}{\partial \varphi} \quad \text { with } \quad\left(B_{1}, B_{2}\right)=(\varphi, 0)
$$

The corresponding conservation laws are given by (2.17):

$$
\begin{gathered}
T_{1}^{t}=\varphi_{t}, \quad T_{1}^{s}=S \varphi_{s}^{-\gamma} \\
T_{2}^{t}=\frac{\varphi_{t}^{2}}{2}+\frac{S}{\gamma-1} \varphi_{s}^{1-\gamma}, \quad T_{2}^{s}=S \varphi_{t} \varphi_{s}^{-\gamma} \\
T_{3}^{t}=\varphi-\varphi_{t} t, \quad T_{3}^{s}=-t S \varphi_{s}^{-\gamma}
\end{gathered}
$$

They represent the conservation of momentum, the conservation of energy and the motion of the center of mass, respectively.

The remaining kernel symmetry operator $X_{4}$ is neither variational nor divergent. Hence, it does not provide any conservation law. 
These conservations laws can be rewritten in the gas dynamics variables as

$$
\begin{gathered}
T_{1}^{t}=u, \quad T_{1}^{s}=S \rho^{\gamma} ; \\
T_{2}^{t}=\frac{u^{2}}{2}+\frac{S}{\gamma-1} \rho^{\gamma-1}, \quad T_{2}^{s}=S \rho^{\gamma} u \\
T_{3}^{t}=\varphi-t u, \quad T_{3}^{s}=-t S \rho^{\gamma} .
\end{gathered}
$$

Notice that the conserved vector $\left(T_{3}^{t}, T_{3}^{s}\right)$ contains the function $\varphi$.

It is possible to present these conservation laws in Eulerian coordinates applying the transformation rule (2.22). We get the conservation law for momentum and energy and the motion of the center of mass as

$$
\begin{gathered}
{ }^{e} T_{1}^{t}=\rho u, \quad{ }^{e} T_{1}^{x}=\rho u^{2}+S \rho^{\gamma} \\
{ }^{e} T_{2}^{t}=\frac{\rho u^{2}}{2}+\frac{S \rho^{\gamma}}{\gamma-1}, \quad{ }^{e} T_{2}^{x}=\left(\frac{\rho u^{2}}{2}+\frac{\gamma S \rho^{\gamma}}{\gamma-1}\right) u \\
{ }^{e} T_{3}^{t}=\rho(x-t u), \quad{ }^{e} T_{3}^{x}=\rho u(x-t u)-t S \rho^{\gamma} .
\end{gathered}
$$

\subsection{Case $\gamma=3$}

For $\gamma=3$ we present only the additional conservation laws. The generic kernel symmetry

$$
Z_{*}=X_{4}=2 t \frac{\partial}{\partial t}+\varphi \frac{\partial}{\partial \varphi}
$$

becomes variational. We also have symmetry (2.30), which is divergent

$$
Z_{* *}=X_{*}=t^{2} \frac{\partial}{\partial t}+t \varphi \frac{\partial}{\partial \varphi} \quad \text { with } \quad\left(B_{1}, B_{2}\right)=\left(\frac{\varphi^{2}}{2}, 0\right) .
$$

The application of Noether's theorem gives the conservation laws of the generic case, which were given in the previous point, and the following two additional conservation laws:

$$
\begin{gathered}
T_{*}^{t}=2 t\left(\frac{\varphi_{t}^{2}}{2}+\frac{S \varphi_{s}^{-2}}{\gamma-1}\right)-\varphi \varphi_{t}, \quad T_{*}^{s}=\left(2 t \varphi_{t}-\varphi\right) S \varphi_{s}^{-3} ; \\
T_{* *}^{t}=t^{2}\left(\frac{\varphi_{t}^{2}}{2}+\frac{S \varphi_{s}^{-2}}{\gamma-1}\right)-t \varphi \varphi_{t}+\frac{\varphi^{2}}{2}, \quad T_{* *}^{s}=\left(t^{2} \varphi_{t}-t \varphi\right) S \varphi_{s}^{-3} .
\end{gathered}
$$

In the gas dynamics variables these conservations laws take the form

$$
\begin{aligned}
T_{*}^{t}=2 t\left(\frac{u^{2}}{2}+\frac{S \rho^{2}}{\gamma-1}\right)-\varphi u, & T_{*}^{s}=(2 t u-\varphi) S \rho^{3} ; \\
T_{* *}^{t}=t^{2}\left(\frac{u^{2}}{2}+\frac{S \rho^{2}}{\gamma-1}\right)-t \varphi u+\frac{\varphi^{2}}{2}, & T_{* *}^{s}=\left(t^{2} u-t \varphi\right) S \rho^{3} .
\end{aligned}
$$

It should be noted that these conserved vectors were presented in [33], where $\varphi$ was considered as a nonlocal variable. 
It is also possible to transform these conservation laws into Eulerian coordinates:

$$
\begin{gathered}
{ }^{e} T_{*}^{t}=2 t\left(\frac{\rho u^{2}}{2}+\frac{S \rho^{3}}{\gamma-1}\right)-x \rho u, \quad{ }^{e} T_{*}^{x}=2 t\left(\frac{\rho u^{2}}{2}+\frac{\gamma S \rho^{3}}{\gamma-1}\right) u-x\left(\rho u^{2}+\rho^{3} S\right) ; \\
{ }^{e} T_{* *}^{t}=t^{2}\left(\frac{\rho u^{2}}{2}+\frac{S \rho^{3}}{\gamma-1}\right)-t x \rho u+\frac{x^{2}}{2} \rho, \\
{ }^{e} T_{* *}^{x}=t^{2}\left(\frac{\rho u^{2}}{2}+\frac{\gamma S \rho^{3}}{\gamma-1}\right) u-t x\left(\rho u^{2}+\rho^{3} S\right)+\frac{x^{2}}{2} \rho u .
\end{gathered}
$$

\section{Conservation laws for special cases of entropy $S(s)$}

There can be additional symmetries admitted by equation (2.12) for some cases of the entropy function $S(s)$. In this section we check whether these additional symmetries lead to additional conservation laws.

\subsection{Isentropic flow $S(s)=A_{0}$}

In Eulerian coordinates the isentropic case is selected out by the differential constraint

$$
S_{x}=0 \text {. }
$$

The admitted generator has the form (2.26) with the coefficients satisfying the equations

$$
\begin{gathered}
(1-\gamma) k_{7}+(\gamma+1) k_{1}-2 k_{3}=0 \\
(\gamma-3) k_{2}=0 .
\end{gathered}
$$

In both cases $\gamma \neq 3$ and $\gamma=3$ we get two symmetries

$$
X_{5}=(\gamma-1) Y_{3}-2 Y_{7}=(\gamma-1) t \frac{\partial}{\partial t}-2 s \frac{\partial}{\partial s}, \quad X_{6}=Y_{8}=\frac{\partial}{\partial s}
$$

in addition to the symmetries given in (2.29) and (2.30). For application of Noether's theorem 22 the study has to be split into the cases $\gamma \neq 3$ and $\gamma=3$.

\subsubsection{Case $\gamma \neq 3$}

The admitted symmetries are given by (2.29) and (4.3). Checking when the symmetry generator

$$
X=\sum_{i=1}^{6} \beta_{i} X_{i}
$$

is variational or divergent by substitution into (2.16), we get the condition

$$
\beta_{4}(\gamma-3)+\beta_{5}(\gamma+1)=0
$$


Therefore, we get two variational symmetries

$$
\begin{array}{r}
Z_{4}=\frac{\gamma+1}{2} X_{4}-\frac{\gamma-3}{2} X_{5}=(3 \gamma-1) t \frac{\partial}{\partial t}+(\gamma-3) s \frac{\partial}{\partial s}+(\gamma+1) \varphi \frac{\partial}{\partial \varphi} \\
Z_{5}=X_{6}=\frac{\partial}{\partial s}
\end{array}
$$

in addition to symmetries (3.2) and (3.3).

Using Noether's theorem, we find conservation laws given in point 3.1 and two conservation laws, corresponding to the additional variational symmetries:

$$
\begin{gathered}
T_{4}^{t}=(3 \gamma-1) t\left(\frac{\varphi_{t}^{2}}{2}+\frac{A_{0}}{\gamma-1} \varphi_{s}^{1-\gamma}\right)+(\gamma-3) s \varphi_{t} \varphi_{s}-(\gamma+1) \varphi \varphi_{t} \\
T_{4}^{s}=(3 \gamma-1) t A_{0} \varphi_{t} \varphi_{s}^{-\gamma}+(\gamma-3) s\left(-\frac{\varphi_{t}^{2}}{2}+\frac{\gamma A_{0}}{\gamma-1} \varphi_{s}^{1-\gamma}\right)-(\gamma+1) A_{0} \varphi \varphi_{s}^{-\gamma} \\
T_{5}^{t}=\varphi_{t} \varphi_{s}, \quad T_{5}^{s}=-\frac{\varphi_{t}^{2}}{2}+\frac{\gamma A_{0}}{\gamma-1} \varphi_{s}^{1-\gamma}
\end{gathered}
$$

These additional conservation laws take the form

$$
\begin{gathered}
T_{4}^{t}=(3 \gamma-1) t\left(\frac{u^{2}}{2}+\frac{A_{0}}{\gamma-1} \rho^{\gamma-1}\right)+(\gamma-3) s \frac{u}{\rho}-(\gamma+1) \varphi u \\
T_{4}^{s}=(3 \gamma-1) t A_{0} u \rho^{\gamma}+(\gamma-3) s\left(-\frac{u^{2}}{2}+\frac{\gamma A_{0}}{\gamma-1} \rho^{\gamma-1}\right)-(\gamma+1) A_{0} \varphi \rho^{\gamma} \\
T_{5}^{t}=\frac{u}{\rho}, \quad T_{5}^{s}=-\frac{u^{2}}{2}+\frac{\gamma A_{0}}{\gamma-1} \rho^{\gamma-1}
\end{gathered}
$$

in the gas dynamics variables.

In Eulerian coordinates the latter conservation law becomes

$$
{ }^{e} T_{5}^{t}=u, \quad{ }^{e} T_{5}^{x}=\frac{u^{2}}{2}+\frac{\gamma A_{0}}{\gamma-1} \rho^{\gamma-1}
$$

The densities of the conserved vector $\left(T_{4}^{t}, T_{4}^{s}\right)$ contain the Lagrangian mass coordinate $s$. For this reason they cannot be rewritten as a local conservation law in Eulerian coordinates.

\subsubsection{Case $\gamma=3$}

We have symmetries (2.29), (2.30) and (4.3). Substituting the generator

$$
X=\sum_{i=1}^{6} \beta_{i} X_{i}+\beta_{*} X_{*}
$$

into (2.16), we get the condition

$$
\beta_{5}=0
$$


Therefore we get only one conservation law in addition to conservation laws given in points 3.1 and 3.2 . It corresponds to the symmetry

$$
Z_{4}=X_{6}=\frac{\partial}{\partial s}
$$

The conserved vector has components

$$
T_{4}^{t}=\varphi_{t} \varphi_{s}, \quad T_{4}^{s}=-\frac{\varphi_{t}^{2}}{2}+\frac{\gamma A_{0}}{\gamma-1} \varphi_{s}^{-2} .
$$

In the gas dynamics variables it takes the form

$$
T_{4}^{t}=\frac{u}{\rho}, \quad T_{4}^{s}=-\frac{u^{2}}{2}+\frac{\gamma A_{0}}{\gamma-1} \rho^{2} .
$$

In Eulerian coordinates we can present it as

$$
{ }^{e} T_{4}^{t}=u, \quad{ }^{e} T_{4}^{x}=\frac{u^{2}}{2}+\frac{\gamma A_{0}}{\gamma-1} \rho^{2} .
$$

\subsection{Entropy $S=A_{0} s^{q}$}

In Eulerian coordinates this case is described by the differential constraint

$$
q \rho S S_{x x}-q \rho S_{x}^{2}-q \rho_{x} S S_{x}+\rho S_{x}^{2}=0 .
$$

One can check that the overdetermined system of equations consisting of the gas dynamics equations and this constraint is involutive.

Calculations show that the admitted generators have the form (2.26) and the coefficients satisfy

$$
\begin{gathered}
(1-\gamma-q) k_{7}+(\gamma+1) k_{1}-2 k_{3}=0 \\
k_{8}=0 \\
(\gamma-3) k_{2}=0
\end{gathered}
$$

In both cases $\gamma \neq 3$ and $\gamma=3$ we get only one additional generator

$$
X_{5}=(1-\gamma-q) Y_{3}+2 Y_{7}=(1-\gamma-q) t \frac{\partial}{\partial t}+2 s \frac{\partial}{\partial s} .
$$

As in the previous case of the entropy function $S(s)$, one needs to study subcases $\gamma=3$ and $\gamma \neq 3$ separately.

\subsubsection{Case $\gamma \neq 3$}

Substituting the generator

$$
X=\sum_{i=1}^{5} \beta_{i} X_{i}
$$

into (2.16), we find the condition for Noether (variational and divergent) symmetries

$$
(3-\gamma) \beta_{4}+(\gamma+q+1) \beta_{5}=0
$$


We get a single additional variational symmetry

$$
\begin{aligned}
Z_{4}=\frac{\gamma+q+1}{2} X_{4} & +\frac{\gamma-3}{2} X_{5} \\
& =(3 \gamma+2 q-1) t \frac{\partial}{\partial t}+(\gamma-3) s \frac{\partial}{\partial s}+(\gamma+1+q) \varphi \frac{\partial}{\partial \varphi}
\end{aligned}
$$

in addition to the symmetries (3.2) and (3.3).

Using Noether's theorem, we compute the components of the conservation law

$$
\begin{aligned}
& T_{4}^{t}=(3 \gamma+2 q-1) t\left(\frac{\varphi_{t}^{2}}{2}+\frac{A_{0} s^{q}}{\gamma-1} \varphi_{s}^{1-\gamma}\right)+(\gamma-3) s \varphi_{t} \varphi_{s}-(\gamma+q+1) \varphi \varphi_{t} \\
& T_{4}^{s}=(3 \gamma+2 q-1) t A_{0} s^{q} \varphi_{t} \varphi_{s}^{-\gamma} \\
&+(\gamma-3) s\left(-\frac{\varphi_{t}^{2}}{2}+\frac{\gamma A_{0} s^{q}}{\gamma-1} \varphi_{s}^{1-\gamma}\right)-(\gamma+q+1) A_{0} s^{q} \varphi \varphi_{s}^{-\gamma}
\end{aligned}
$$

In the gas dynamics variables we get

$$
\begin{array}{r}
T_{4}^{t}=(3 \gamma+2 q-1) t\left(\frac{u^{2}}{2}+\frac{A_{0} s^{q}}{\gamma-1} \rho^{\gamma-1}\right)+(\gamma-3) s \frac{u}{\rho}-(\gamma+q+1) \varphi u, \\
T_{4}^{s}=(3 \gamma+2 q-1) t A_{0} s^{q} u \rho^{\gamma} \\
+(\gamma-3) s\left(-\frac{u^{2}}{2}+\frac{\gamma A_{0} s^{q}}{\gamma-1} \rho^{\gamma-1}\right)-(\gamma+q+1) A_{0} s^{q} \varphi \rho^{\gamma} .
\end{array}
$$

To rewrite this conservation law in Eulerian coordinates we use the transformation rule (2.22) and

$$
s=q \rho \frac{S}{S_{x}}
$$

We obtain

$$
\begin{gathered}
{ }^{e} T_{4}^{t}=(3 \gamma+2 q-1) t\left(\frac{\rho u^{2}}{2}+\frac{S}{\gamma-1} \rho^{\gamma}\right)+(\gamma-3) q \rho u \frac{S}{S_{x}}-(\gamma+q+1) x \rho u \\
{ }^{e} T_{4}^{x}=(3 \gamma+2 q-1) t\left(\frac{\rho u^{2}}{2}+\frac{\gamma S}{\gamma-1} \rho^{\gamma}\right) u \\
+(\gamma-3) q \rho \frac{S}{S_{x}}\left(\frac{u^{2}}{2}+\frac{\gamma S}{\gamma-1} \rho^{\gamma-1}\right)-(\gamma+q+1) x\left(\rho u^{2}+S \rho^{\gamma}\right) .
\end{gathered}
$$

\subsubsection{Case $\gamma=3$}

For symmetries (2.29), (2.30) and (4.18) substitution of the generator

$$
X=\sum_{i=1}^{5} \beta_{i} X_{i}+\beta_{*} X_{*}
$$

into (2.16) gives the condition

$$
(q+4) \beta_{5}=0
$$


for variational and divergent symmetries. There are two possibilities.

a) For $q \neq-4$ there is no additional generators. We get the conservation laws which were described in points 3.1 and 3.2 .

b) If $q=-4$, there is one additional variational symmetry

$$
Z_{4}=\frac{1}{2} X_{5}=t \frac{\partial}{\partial t}+s \frac{\partial}{\partial s}
$$

The corresponding conservation law has densities

$$
\begin{aligned}
T_{4}^{t}=t\left(\frac{\varphi_{t}^{2}}{2}+\frac{A_{0} s^{q}}{\gamma-1} \varphi_{s}^{1-\gamma}\right) & +s \varphi_{t} \varphi_{s} \\
T_{4}^{s} & =t A_{0} s^{q} \varphi_{t} \varphi_{s}^{-\gamma}+s\left(-\frac{\varphi_{t}^{2}}{2}+\frac{\gamma A_{0} s^{q}}{\gamma-1} \varphi_{s}^{1-\gamma}\right) .
\end{aligned}
$$

In the gas dynamics variables we get

$$
\begin{aligned}
T_{4}^{t}=t\left(\frac{u^{2}}{2}+\frac{A_{0} s^{q}}{\gamma-1} \rho^{\gamma-1}\right) & +s \frac{u}{\rho}, \\
& T_{4}^{s}=t A_{0} s^{q} u \rho^{\gamma}+s\left(-\frac{u^{2}}{2}+\frac{\gamma A_{0} s^{q}}{\gamma-1} \rho^{\gamma-1}\right) .
\end{aligned}
$$

These components get transformed into Eulerian coordinates as

$$
\begin{aligned}
&{ }^{e} T_{4}^{t}=t\left(\frac{\rho u^{2}}{2}+\frac{S}{\gamma-1} \rho^{\gamma}\right)+q \rho u \frac{S}{S_{x}} \\
&{ }^{e} T_{4}^{x}=\left(t \rho u+q \rho \frac{S}{S_{x}}\right)\left(\frac{u^{2}}{2}+\frac{\gamma S}{\gamma-1} \rho^{\gamma-1}\right) .
\end{aligned}
$$

\subsection{Entropy $S(s)=A_{0} e^{q s}$}

This assumption for the entropy is given in Eulerian coordinates by the differential constraint

$$
S_{x}=\rho q S
$$

It is possible to check that the overdetermined system of the gas dynamics equations and the latter constraint is involutive.

The classifying equations (2.28) lead to

$$
\begin{gathered}
(\gamma+1) k_{1}-2 k_{3}-q k_{8}=0 \\
k_{7}=0 \\
(\gamma-3) k_{2}=0
\end{gathered}
$$

For both cases $\gamma=3$ and $\gamma \neq 3$ we find one additional symmetry:

$$
X_{5}=q Y_{3}-2 Y_{8}=q t \frac{\partial}{\partial t}-2 \frac{\partial}{\partial s} .
$$




\subsubsection{Case $\gamma \neq 3$}

Substitution of the generator

$$
X=\sum_{i=1}^{5} \beta_{i} X_{i}
$$

into (2.16) gives that the condition for Noether symmetries as

$$
(3-\gamma) \beta_{4}-q \beta_{5}=0
$$

There is the variational symmetry

$$
Z_{4}=\frac{q}{2} X_{4}+\frac{3-\gamma}{2} X_{5}=2 q t \frac{\partial}{\partial t}+(\gamma-3) \frac{\partial}{\partial s}+q \varphi \frac{\partial}{\partial \varphi}
$$

in addition to the symmetries (3.2) and (3.3), which gives the conserved vector

$$
\begin{aligned}
& T_{3}^{t}=2 q t\left(\frac{\varphi_{t}^{2}}{2}+\frac{A_{0} e^{q s}}{\gamma-1} \varphi_{s}^{1-\gamma}\right)-q \varphi \varphi_{t}+(\gamma-3) \varphi_{t} \varphi_{s} \\
& T_{3}^{s}=2 q t A_{0} e^{q s} \varphi_{s}^{-\gamma} \varphi_{t}-q A_{0} e^{q s} \varphi \varphi_{s}^{-\gamma}+(\gamma-3)\left(-\frac{\varphi_{t}^{2}}{2}+\frac{\gamma A_{0} e^{q s}}{\gamma-1} \varphi_{s}^{1-\gamma}\right) .
\end{aligned}
$$

It can be rewritten in the gas dynamics variables as

$$
\begin{aligned}
& T_{3}^{t}=2 q t\left(\frac{u^{2}}{2}+\frac{A_{0} e^{q s}}{\gamma-1} \rho^{\gamma-1}\right)-q \varphi u+(\gamma-3) \frac{u}{\rho} \\
& T_{3}^{s}=2 q t A_{0} e^{q s} \rho^{\gamma} u-q A_{0} e^{q s} \varphi \rho^{\gamma}+(\gamma-3)\left(-\frac{u^{2}}{2}+\frac{\gamma A_{0} e^{q s}}{\gamma-1} \rho^{\gamma-1}\right) .
\end{aligned}
$$

We can also give this conservation law in Eulerian coordinates

$$
\begin{gathered}
{ }^{e} T_{3}^{t}=2 q t\left(\frac{\rho u^{2}}{2}+\frac{A_{0} e^{q s}}{\gamma-1} \rho^{\gamma}\right)-q x \rho u+(\gamma-3) u \\
{ }^{e} T_{3}^{x}=2 q t\left(\frac{\rho u^{2}}{2}+\frac{\gamma A_{0} e^{q s}}{\gamma-1} \rho^{\gamma}\right) u-q x\left(\rho u^{2}+A_{0} e^{q s} \rho^{\gamma}\right)+(\gamma-3)\left(\frac{u^{2}}{2}+\frac{\gamma A_{0} e^{q s}}{\gamma-1} \rho^{\gamma-1}\right) .
\end{gathered}
$$

\subsubsection{Case $\gamma=3$}

In this case we consider symmetries (2.29), (2.30) and (4.32). Looking for Noether symmetries, we substitute

$$
X=\sum_{i=1}^{5} \beta_{i} X_{i}+\beta_{*} X_{*}
$$

into (2.16) and obtain gives

$$
q \beta_{5}=0 .
$$

There are no additional Noether symmetries. Therefore we do not any conservation laws in addition to those given in points 3.1 and 3.2 . 


\subsection{Discussion}

First of all we note that to select variational or divergent symmetries using property (2.16) of an admitted symmetry it was important to consider a generator $X$ in its general form, i.e. as a linear combination of all admitted symmetries.

Complete Lie group classification of the gas dynamics equation in the Lagrangian coordinates (2.12) allows us to find all conservation laws which can be found using Noether's theorem and admitted symmetries. The group classification has three cases of the entropy for which there exist additional symmetries. In Eulerian coordinates these three cases are defined by differential constraints of first or second order. Notice that the overdetermined systems which consist of the gas dynamics equations and one of the considered differential constraints are involutive. The authors of [36, 35] also found conservation laws corresponding to special forms of the entropy. Here the symmetry nature of these conservation laws is explained.

In contrast to 33 the conservation laws, obtained in this paper, are local. It should be also noted that this conservation laws are naturally derived: their counterparts in Lagrangian coordinates were derived directly using Noether's theorem without any additional assumptions. We should also mention that in contrast to two-dimensional Lagrangian gas dynamics the special cases of the entropy in Lagrangian coordinates are given explicitly. In the two-dimensional case [18] the entropy is arbitrary, but the admitted symmetry operators contain functions satisfying quasilinear partial differential equations.

\section{Finite-difference models}

\subsection{Invariance and Euler coordinates}

The first problem in discretization of differential equations is the choice of difference mesh. For discretization of the gas dynamics system (2.1a), (2.1b), (2.4), which is given in Euler coordinates, the simplest choice seems to be an orthogonal mesh in $(t, x)$ plane. However, this mesh is not invariant that destroys invariance of difference equations considered on such mesh. Indeed, as it was shown in [9, 14] the necessary condition for a mesh to preserve its invariance under a group transformation generated by the operator

$$
X=\xi^{t} \frac{\partial}{\partial t}+\xi^{x} \frac{\partial}{\partial x}+\ldots
$$

is the following:

$$
D_{+h}\left(\xi^{t}\right)=-D_{+\tau}\left(\xi^{x}\right),
$$

where $D_{+h}$ and $D_{+\tau}$ are the operators of difference differentiation in $x$ and $t$ directions respectively.

The system (2.1a), (2.1b), (2.4) admits 24] the 6-parameter Lie symmetry group of point transformations that corresponds to the following Lie algebra of 
infinitesimal operators:

$$
\begin{array}{rlrl}
X_{1}=\frac{\partial}{\partial t}, & X_{2}=\frac{\partial}{\partial x}, & X_{3}=t \frac{\partial}{\partial t}+x \frac{\partial}{\partial x}, & X_{4}=t \frac{\partial}{\partial x}+\frac{\partial}{\partial u} \\
X_{5}=x \frac{\partial}{\partial x}+u \frac{\partial}{\partial u}-2 \rho \frac{\partial}{\partial \rho}, & X_{6}=\rho \frac{\partial}{\partial \rho}+p \frac{\partial}{\partial p} .
\end{array}
$$

In the special case $\gamma=3$ there is one more symmetry

$$
X_{7}=t^{2} \frac{\partial}{\partial t}+t x \frac{\partial}{\partial x}+(x-t u) \frac{\partial}{\partial u}-t \rho \frac{\partial}{\partial \rho}-3 t p \frac{\partial}{\partial p} .
$$

It is easy to see, the Galileo transformation, given by the operator $X_{4}$, does not satisfy the criterion (5.2). The same is true for $X_{7}$. It means that one should look for an invariant moving mesh in Eulerian coordinates.

To obtain an invariant moving mesh we chose the following difference stencil with two time layers:

- independent variables:

$$
t=t_{j}, \quad \hat{t}=t_{j+1} ; \quad x=x_{i}^{j}, \quad x_{+}=x_{i+1}^{j}, \quad \hat{x}=x_{i}^{j+1}, \quad \hat{x}_{+}=x_{i+1}^{j+1} ;
$$

- dependent variables in the nodes of the mesh (as $x)$ :

$$
u, \quad u_{+}, \quad \hat{u}, \quad \hat{u}_{+} ; \quad \rho, \quad \rho_{-}, \quad \hat{\rho}, \quad \hat{\rho}_{-} ; \quad p, \quad p_{-}, \quad \hat{p}, \quad \hat{p}_{-} .
$$

Then, we find the finite-difference invariants for symmetries (5.3) as the solutions of system of linear equations

$$
X_{i} I\left(t, \hat{t}, x, x_{+}, \hat{x}, \hat{x}_{+}, \ldots, \rho, \rho_{-}, \hat{p}, \hat{p}_{-}\right)=0, \quad i=1, \ldots, 6 .
$$

Here we assume that the operator is prolonged on all variables of the stencil. There are 12 functionally independent invariants

$$
\begin{aligned}
& \frac{\hat{h}_{+}}{h_{+}}, \quad \frac{\tau}{h_{+}} \sqrt{\frac{p}{\rho}}, \quad \sqrt{\frac{\rho}{p}}\left(\frac{\hat{x}-x}{\tau}-u\right), \\
& \sqrt{\frac{\rho}{p}}\left(u_{+}-u\right), \quad \sqrt{\frac{\rho}{p}}(\hat{u}-u), \quad \sqrt{\frac{\rho}{p}}\left(\hat{u}_{+}-\hat{u}\right), \\
& \frac{p_{+}}{p}, \quad \frac{\hat{p}}{p}, \quad \frac{\hat{p}_{+}}{\hat{p}}, \quad \frac{\hat{\rho}}{\rho}, \quad \frac{\hat{\rho}_{+}}{\hat{\rho}}, \quad \frac{\rho_{+}}{\hat{\rho}},
\end{aligned}
$$

where $\tau=\hat{t}-t, h_{+}=x_{+}-x$ and $\hat{h}_{+}=\hat{x}_{+}-\hat{x}$.

These invariants suggest, for example, an invariant moving mesh given by

$$
\sqrt{\frac{\rho}{p}}\left(\frac{\hat{x}-x}{\tau}-u\right)=0
$$

or, equivalently,

$$
\frac{\hat{x}-x}{\tau}=u
$$


In the continuous limit it corresponds to the evolution of the spacial variable $x$ given as

$$
\frac{d x}{d t}=u
$$

Thus, we arrive at choosing the mass Lagrangian coordinates.

Below we introduce a scheme, which is invariant with respect to symmetries of $1+1 \mathrm{D}$ gas dynamics in the case $\gamma \neq 3$.

\subsection{Popov-Samarskii scheme}

One of the best known numerical schemes for one-dimensional gas dynamics in the mass mass coordinates is completely conservative scheme introduced by Yu. P. Popov and A. A. Samarskii [27, 29]. It was derived as a scheme which preserves conservation laws of mass, momentum and energy. Usually it is given for gas dynamics equations in the form

$$
\begin{gathered}
\left(\frac{1}{\rho}\right)_{t}=u_{s}, \\
u_{t}+p_{s}=0, \\
\varepsilon_{t}=-p u_{s}, \\
x_{t}=u,
\end{gathered}
$$

where

$$
\varepsilon=\frac{p}{(\gamma-1) \rho}, \quad \gamma \neq 0,1
$$

is the internal energy of the gas. For the polytropic gas equation (5.10c) is equivalent to (2.8c) and (2.9). However, the scheme can also be used for other equations of the internal energy $\varepsilon=\varepsilon(\rho, p)$.

The gas dynamics system is presented with the help of the internal energy $\varepsilon$ to emphasize importance of the relation

$$
\varepsilon_{t}=-p\left(\frac{1}{\rho}\right)_{t}
$$

for qualitatively correct discretization. This relation shows that the change of the internal energy happens due to the work done by the pressure forces.

\subsubsection{Notations}

Let us introduce notations which are needed to present the scheme. In the coordinates $(t, s)$ we can consider an orthogonal mesh. For simplicity we chose a uniform mesh for the Lagrange mass variable $s$, i.e. neighbouring spatial step

$$
h^{s}=s_{i+1}-s_{i} \quad \text { and } \quad h_{-}^{s}=s_{i}-s_{i-1}
$$

will be equal: $h^{s}=h_{-}^{s}$. Of course, it is also possible to consider nonuniform steps $h_{i}^{s}=s_{i+1}-s_{i}$.

Variables $u$ and $x$ are taken in the nodes of the mesh as

$$
u=u_{i}^{j}, \quad u_{+}=u_{i+1}^{j}, \quad \hat{u}=u_{i}^{j+1}, \quad \hat{u}_{+}=u_{i+1}^{j+1} .
$$


The time derivative and forward spatial derivatives will be

$$
u_{t}=\frac{\hat{u}-u}{\tau}, \quad u_{s}=\frac{u_{i+1}^{j}-u_{i}^{j}}{s_{i+1}-s_{i}}=\frac{u_{+}-u}{h^{s}} .
$$

Variables $\rho, p$ and $\varepsilon$ are assigned to the midpoints $i-1 / 2, i+1 / 2, i+3 / 2$ and so on. For example,

$$
\rho_{-}=\rho_{i-1 / 2}^{j}, \quad \rho=\rho_{i+1 / 2}^{j}, \quad \rho_{+}=\rho_{i+3 / 2}^{j} .
$$

For their backward spatial derivatives we take

$$
p_{\bar{s}}=\frac{p_{i+1 / 2}^{j}-p_{i-1 / 2}^{j}}{h^{s}},
$$

For pressure $p$ we will need the linear interpolation value for the nodes of the mesh. For example, for the node $\left(t_{j}, s_{i}\right)$ we have

$$
p_{*}=p_{* i}^{j}=\frac{p_{i-1 / 2}^{j}+p_{i+1 / 2}^{j}}{2} .
$$

Weighted values for all variables will be denoted as

$$
y^{(\alpha)}=\alpha \hat{y}+(1-\alpha) y, \quad 0 \leq \alpha \leq 1 .
$$

\subsubsection{The scheme and its properties}

Popov-Samaskii scheme [27]

$$
\begin{gathered}
\left(\frac{1}{\rho}\right)_{t}=\left(u^{(0.5)}\right)_{s} \\
u_{t}=-p_{\bar{s}}^{(\alpha)}, \\
\varepsilon_{t}=-p^{(\alpha)}\left(u^{(0.5)}\right)_{s}, \\
x_{t}=u^{(0.5)} .
\end{gathered}
$$

is invariant with respect to symmetries (5.3) extended to varaible $s$ :

$$
\begin{gathered}
X_{1}=\frac{\partial}{\partial t}, \quad X_{2}=\frac{\partial}{\partial x}, \quad X_{3}=t \frac{\partial}{\partial t}+s \frac{\partial}{\partial s}+x \frac{\partial}{\partial x}, \quad X_{4}=t \frac{\partial}{\partial x}+\frac{\partial}{\partial u}, \\
X_{5}=-s \frac{\partial}{\partial s}+x \frac{\partial}{\partial x}+u \frac{\partial}{\partial u}-2 \rho \frac{\partial}{\partial \rho}, \quad X_{6}=s \frac{\partial}{\partial s}+\rho \frac{\partial}{\partial \rho}+p \frac{\partial}{\partial p} .
\end{gathered}
$$

It also admit translations of the new dependent variable $s$

$$
X_{7}=\frac{\partial}{\partial s} \text {. }
$$

However, it is not invariant for the projective operator

$$
X_{8}=t^{2} \frac{\partial}{\partial t}+(x-t u) \frac{\partial}{\partial u}-t \rho \frac{\partial}{\partial \rho}-3 t p \frac{\partial}{\partial p}+t x \frac{\partial}{\partial x},
$$

which is admitted by (5.10), (5.11) in the special case $\gamma=3$.

Let us discuss the properties of this scheme. In the general case $\varepsilon(\rho, p)$, i.e. not only for the ideal gas (5.11), the scheme (5.20) has the following conservation laws: 
1. Conservation of mass (5.20a $)$, which manifests the consistency of the relations

$$
\frac{h_{i}^{s}}{\rho_{i+1 / 2}^{j}}=x_{i+1}^{j}-x_{i}^{j}, \quad \frac{h_{i}^{s}}{\rho_{i+1 / 2}^{j+1}}=x_{i+1}^{j+1}-x_{i}^{j+1}
$$

with the evolution equation (5.20d).

2. Conservation of momentum

$$
[u]_{t}+\left[p^{(\alpha)}\right]_{\bar{s}}=0 .
$$

3. Conservation of energy

$$
\left[\varepsilon+\frac{u^{2}+u_{+}^{2}}{4}\right]_{t}+\left[p_{*}^{(\alpha)} u^{(0.5)}\right]_{s}=0 .
$$

4. The law for the center of mass motion

$$
[x-t u]_{t}-\left[t^{(0.5)} p^{(\alpha)}\right]_{\bar{s}}=0,
$$

which was not mentioned by the authors [27].

These discrete conservation laws correspond to continuous conservation laws for

1. mass

$$
\left[\frac{1}{\rho}\right]_{t}-[u]_{s}=0
$$

2. momentum

$$
[u]_{t}+[p]_{s}=0
$$

3. energy

$$
\left[\frac{u^{2}}{2}+\frac{p}{(\gamma-1) \rho}\right]_{t}+[p u]_{s}=0
$$

4. center of mass motion

$$
[x-t u]_{t}-[t p]_{s}=0 .
$$

For the polytropic gas (5.11) with $\gamma=3$ there exist two additional conservation laws

$$
\begin{array}{r}
{\left[2 t\left(\frac{u^{2}}{2}+\frac{p}{(\gamma-1) \rho}\right)-x u\right]_{t}+[2 t p u-x p]_{s}=0} \\
{\left[t^{2}\left(\frac{u^{2}}{2}+\frac{p}{(\gamma-1) \rho}\right)-t x u+\frac{x^{2}}{2}\right]_{t}+\left[t^{2} p u-t x p\right]_{s}=0}
\end{array}
$$

which the scheme (5.20) does not have. 
The scheme does not preserve entropy, or

$$
S=\frac{p}{\rho^{\gamma}}=\text { const }
$$

for the streamlines of the flow. However, it holds the relation

$$
\frac{\Delta p}{p^{(\alpha)}}=\gamma \frac{\Delta \rho}{\rho^{(\alpha)}}
$$

where $\Delta p=\hat{p}-p$ and $\Delta \rho=\hat{\rho}-\rho$. This discrete relation approximates its differential counterpart

$$
\frac{p_{t}}{p}=\gamma \frac{\rho_{t}}{\rho}
$$

with the order $0(\tau)$. Thus, we get an error for the entropy evolution.

It should also be noted that the scheme (5.20) has the relation

$$
\varepsilon_{t}=-p^{(\alpha)}\left(\frac{1}{\rho}\right)_{t}
$$

which is a qualitatively correct discretization of the continuous case relation (5.12). It represents a correct balance of the internal energy.

\subsection{Invariance of finite-difference schemes}

Let us show how one can use invariant to construct finite-difference schemes. For the case $\gamma \neq 3$ we will show how the scheme, given in the previous point, can be expressed in term of the invariants. For the case $\gamma=3$ we will suggest an invariant scheme.

\subsubsection{Case $\gamma \neq 3$}

We consider an orthogonal mesh in $(t, s)$ coordinates and specify the stencil variables as

- independent variables:

$$
t=t_{j}, \quad \hat{t}=t_{j+1}, \quad s=s_{i}, \quad s_{+}=s_{i+1}, \quad s_{-}=s_{i-1}
$$

- dependent variables in the nodes of the mesh:

$$
u=u_{i}^{j}, \quad u_{+}=u_{i+1}^{j}, \quad \hat{u}=u_{i}^{j+1}, \quad \hat{u}_{+}=u_{i+1}^{j+1}, \quad x, \quad x_{+}, \quad \hat{x}, \quad \hat{x}_{+} ;
$$

- dependent variables in the midpoints of the mesh:

$$
\rho=\rho_{i}^{j}, \quad \rho_{-}=\rho_{i-1}^{j}, \quad \hat{\rho}=\rho_{i}^{j+1}, \quad \hat{\rho}_{-}=\rho_{i-1}^{j+1}, \quad p, \quad p_{-}, \quad \hat{p}, \quad \hat{p}_{-} .
$$

In the space of 21 stencil variables there are 14 invariants for 7 symmetries (5.21), (5.22):

$$
I_{1}=\frac{h_{-}^{s}}{h^{s}}, \quad I_{2}=\frac{\tau}{h^{s}} \sqrt{\rho p}, \quad I_{3}=\sqrt{\frac{\rho}{p}}\left(\frac{\hat{x}-x}{\tau}-u\right), \quad I_{4}=\sqrt{\frac{\rho}{p}}\left(\frac{\hat{x}-x}{\tau}-\hat{u}\right),
$$




$$
\begin{gathered}
I_{5}=\sqrt{\frac{\rho}{p}}\left(u_{+}-u\right), \quad I_{6}=\sqrt{\frac{\rho}{p}}\left(\hat{u}_{+}-\hat{u}\right), \quad I_{7}=\frac{\rho\left(x_{+}-x\right)}{h^{s}}, \quad I_{8}=\frac{\hat{\rho}\left(\hat{x}_{+}-\hat{x}\right)}{h^{s}}, \\
I_{9}=\frac{\rho_{-}}{\rho}, \quad I_{10}=\frac{\hat{\rho}}{\rho}, \quad I_{11}=\frac{\hat{\rho}_{-}}{\hat{\rho}}, \quad I_{12}=\frac{p_{-}}{p}, \quad I_{13}=\frac{\hat{p}}{p}, \quad I_{14}=\frac{\hat{p}_{-}}{\hat{p}} .
\end{gathered}
$$

One can find the scheme (5.20) approximating the gas dynamics system (5.10) with the help of these invariants as

$$
\begin{gathered}
\frac{1}{I_{10}}-1=I_{2} \frac{I_{5}+I_{6}}{2}, \\
I_{3}-I_{4}=-I_{2}\left(\alpha\left(I_{13}-I_{13} I_{14}\right)+(1-\alpha)\left(1-I_{12}\right)\right), \\
\frac{1}{\gamma-1}\left(\frac{I_{13}}{I_{10}}-1\right)=-I_{2}\left(\alpha I_{13}+(1-\alpha)\right) \frac{I_{5}+I_{6}}{2}, \\
I_{3}+I_{4}=0 .
\end{gathered}
$$

\subsubsection{Case $\gamma=3$}

For the case $\gamma=3$ we can suggest invariant schemes, but have difficulties to find invariant schemes with conservation laws.

In comparison to the previous point 5.3 .1 we have one more symmetry, namely (5.23). Therefore, we get one invariant less.

There obtain 13 invariants

$$
\begin{aligned}
& J_{1}=\frac{h_{-}^{s}}{h^{s}}, \quad J_{2}=\frac{\tau}{h^{s}}(\rho p \hat{\rho} \hat{p})^{\frac{1}{4}}, \quad J_{3}=\sqrt{\frac{\rho}{p}}\left(\frac{\hat{x}-x}{\tau}-u\right), \quad J_{4}=\sqrt{\frac{\hat{\rho}}{\hat{p}}}\left(\frac{\hat{x}-x}{\tau}-\hat{u}\right), \\
& J_{5}=\sqrt{\frac{\rho}{p}}\left(\frac{h_{+}}{\tau}+u_{+}-u\right), \quad J_{6}=\sqrt{\frac{\hat{\rho}}{\hat{p}}}\left(-\frac{\hat{h}_{+}}{\tau}+\hat{u}_{+}-\hat{u}\right), \quad J_{7}=\frac{\rho\left(x_{+}-x\right)}{h^{s}}, \\
& J_{8}=\frac{\hat{\rho}\left(\hat{x}_{+}-\hat{x}\right)}{h^{s}}, \quad J_{9}=\frac{\hat{p}}{p}\left(\frac{\rho}{\hat{\rho}}\right)^{3}, \quad J_{10}=\frac{\rho_{-}}{\rho}, \quad J_{11}=\frac{\hat{\rho}_{-}}{\hat{\rho}}, \quad J_{12}=\frac{p_{-}}{p}, \quad J_{13}=\frac{\hat{p}_{-}}{\hat{p}} .
\end{aligned}
$$

There are many possibilities to approximate the gas dynamics system (5.10), (5.11) with the help of these invariants. Let us suggest the following explicit invariant scheme

$$
\begin{gathered}
\hat{\rho}\left(\hat{x}_{+}-\hat{x}\right)=\rho\left(x_{+}-x\right), \\
\frac{\hat{u}-u}{\tau}=-\left(\frac{\hat{\rho}}{\rho}\right)^{2} \frac{p-p_{-}}{h^{s}}, \\
\frac{\hat{p}}{\hat{\rho}^{3}}=\frac{p}{\rho^{3}} \\
\frac{\hat{x}-x}{\tau}=u
\end{gathered}
$$

In term of the invariants this scheme is written as

$$
J_{7}=J_{8}
$$




$$
\begin{gathered}
J_{4}=J_{2} J_{9}^{-3 / 4}\left(1-J_{12}\right), \\
J_{9}=1, \\
J_{3}=0 .
\end{gathered}
$$

The scheme keeps an entropy along streamlines (5.34) and possesses conservation of mass (5.38a). Note that the first equation can be rewritten as

$$
\frac{1}{\tau}\left(\frac{1}{\hat{\rho}}-\frac{1}{\rho}\right)=\frac{u_{+}-u}{h^{s}} .
$$

Let us note that implicit invariant schemes are also possible.

\section{Concluding remarks}

In the present paper there were considered Lie point symmetries of the onedimensional gas dynamics equations of the polytropic gas in Lagrangian coordinates. Complete Lie group classification of these equations reduced to the scalar second-order PDE is performed. The classification parameter is the entropy. It was shown that for the isentropic gas and for the entropy depending of mass coordinate as power and exponential law there exist extensions of the symmetry properties. Therefore, there are additional conservation laws. For these cases Noether's theorem was applied for constructing conservation laws for the scalar second-order PDE. The conservation laws were later represented in the gas dynamics variables.

For the basic adiabatic case invariant and conservative difference schemes were discussed. The whole set of difference invariants was constructed for the general case and for the extension case $\gamma=3$. It was shown that the PopovSamarskii scheme is invariant for the basic case symmetries, but does not allow the additional symmetry which exists for $\gamma=3$. This scheme possesses the whole set of conservation laws, excluding two additional laws which hold for the exceptional value of $\gamma$. It also does not preserve the entropy along streamlines. The scheme which preserve all symmetries for $\gamma=3$ was developed. This scheme holds exactly the entropy along the streamlines but has only one conservation law, namely the conservation laws of mass.

\section{Acknowledgements}

The research was supported by Russian Science Foundation Grant No 18-1100238 'Hydrodynamics-type equations: symmetries, conservation laws, invariant difference schemes'.

\section{References}

[1] I. S. Akhatov, R. K. Gazizov and N. H. Ibragimov, Nonlocal symmetries. Heuristic approach., J. Math. Sci., 55(1) 1401-1450, 1991. 
[2] W. F. Ames, R. L. Anderson, V. A. Dorodnitsyn, E. V. Ferapontov, R. K. Gazizov, N. H. Ibragimov and S. R. Svirshchevskii, CRC Handbook of Lie Group Analysis of Differential Equations, Volume I: Symmetries, Exact Solutions and Conservation Laws, CRC Press, 1994.

[3] V. K. Andreev, O. V. Kaptsov, V. V. Pukhnachov and A. A. Rodionov, Applications of Group-Theoretic Methods in Hydrodynamics, Kluwer, Dordrecht, 1998.

[4] M. Bakirova, V. Dorodnitsyn and R. Kozlov, Invariant difference schemes for heat transfer equations with a source, J. Phys. A: Math. Gen., 30, 8139 (1997)

[5] G. W. Bluman, A. F. Cheviakov and S. C. Anco, Applications of Symmetry Methods to Partial Differential Equations, Springer, New York, 2010. Applied Mathematical Sciences, Vol.168.

[6] B. J. Cantwell, Introduction to symmetry analysis, Cambridge University Press, Cambridge, 2002.

[7] G. G. Chernyi, Gas dynamics, Nauka, Moscow, 1988, (in Russian).

[8] B. Després and C. Mazeran, Lagrangian gas dynamics in two dimensions and Lagrangian systems, Arch. Rational Mech. Anal., 178 327-372, 2005 .

[9] V. A. Dorodnitsyn, Transformation groups in a space of net variables, in VINITI Acad. Sci. USSR, Itogi Nauki i Techniki, 34, 149-190 (1989), (in Russian); see English translation in J. Sov. Math. 55, 1490 (1991).

[10] V. A. Dorodnitsyn, Finite-difference analog of the Noether theorem, Dokl. Akad. Nauk, 328, 678, 1993 (in Russian).

[11] V. Dorodnitsyn, R. Kozlov and P. Winternitz, Lie group classification of second-order ordinary difference equations, J. Math. Phys., 41 480-504, 2000 .

[12] V. Dorodnitsyn and R. Kozlov, A heat transfer with a source: the complete set of invariant difference schemes, J. of Nonlinear Math. Physics, 10 16-50, 2003.

[13] V. Dorodnitsyn, R. Kozlov and P. Winternitz, Continuous symmetries of Lagrangians and exact solutions of discrete equations, J. of Math. Physics, 45 336-359, 2004.

[14] V. Dorodnitsyn, Applications of Lie Groups to Difference Equations, Chapman \& Hall/CRC differential and integral equations series, 2011.

[15] N. H. Ibragimov, editor, CRC Handbook of Lie Group Analysis of Differential Equations, volume 2, CRC Press, Boca Raton, 1995. 
[16] N. H. Ibragimov. Elementary Lie Group Analysis and Ordinary Differential Equations, Wiley \& Sons, Chichester, 1999.

[17] N. H. Ibragimov, A new conservation theorem, J. Math. Anal. Appl., 333 311-328, 2007.

[18] E. I. Kaptsov and S. V. Meleshko, Conservation Laws of the TwoDimensional Gas Dynamics Equations, submitted to International Journal of Non-Linear Mechanics.

[19] D. Levi and P. Winternitz, Continuous symmetries of difference equations, J. Phys. A: Math. Gen., 39 1-63, 2006

[20] J. Marsden and T. Ratiu, Introduction to Mechanics and Symmetry, Springer-Verlag, New York, 1994.

[21] S. V. Meleshko, Methods for Constructing Exact Solutions of Partial Differential Equations, Mathematical and Analytical Techniques with Applications to Engineering, Springer, New York, 2005.

[22] E. Noether. Invariante Variationsprobleme, Nachr. d. Königlichen Gesellschaft der Wissenschaften zu Göttingen, Math-phys. Klasse, pages 235-257, 1918. English translation in: Transport Theory and Statistical Physics, vol. 1, No. 3, 1971, 186-207 arXiv:physics/0503066 [physics.hist-ph]).

[23] P. J. Olver, Applications of Lie groups to differential equations, SpringerVerlag, New York, 1986.

[24] L. V. Ovsiannikov, Group analysis of differential equations, Nauka, Moscow, 1978. English translation, W. F. Ames, Ed., published by Academic Press, New York, 1982.

[25] L. V. Ovsiannikov, Program SUBMODELS. Gas dynamics. J. Appl. Maths Mechs., 58(4) 30-55, 1994.

[26] L. V. Ovsiannikov, Lectures on the gas dynamics equations, Institute of computer studies, Moscow, Izhevsk, 2003, Second edition.

[27] Yu. P. Popov and A. A. Samarskii, Completely conservative difference schemes Zh. Vychisl. Mat. Mat. Fiz., 9 953-58, 1969.

[28] B. L. Rozhdestvenskii and N. N. Yanenko, Systems of quasilinear equations and their applications to gas dynamics, 2nd ed., Nauka, Moscow, 1978. English translation published by Amer. Math. Soc., Providence, RI, 1983.

[29] A. A. Samarskii and Yu. P. Popov, Finite-difference schemes of gasdynamics, Nauka, Moscow, 1975 (in Russian).

[30] R. L. Seliger and G. B. Whitham, Variational principles in continuum mechanics, Proc. R. Soc. London. A, 305 1-25, 1968. 
[31] Yu. D. Shmyglevski, Analytical study of gas dynamics and fluid, Editorial URSS, Moscow, 1999, in Russian.

[32] P. Siriwat, C. Kaewmanee and S. V. Meleshko, Symmetries of the hyperbolic shallow water equations and the Green-Naghdi model in Lagrangian coordinates, International Journal of Non-Linear Mechanics, 86 185-195, 2016.

[33] A. Sjöberg and F. M. Mahomed, Non-local symmetries and conservation laws for one-dimensional gas dynamics equations. Applied Mathematics and Computation, 150 379397, 2004.

[34] P. Voraka, C. Kaewmanee and S. V. Meleshko. Symmetries of the shallow water equations in the Boussinesq approximation, Commun. Nonlinear Sci. Numer. Simulat., 67 1-12, 2019.

[35] G. Webb, Magnetohydrodynamics and Fluid Dynamics: Action Principles and Conservation Laws, Springer, Heidelberg, 2018. Lecture Notes in Physics, v. 946.

[36] G. M. Webb and G. P. Zank, Scaling symmetries, conservation laws and action principles in one-dimensional gas dynamics, J. Phys. A: Math. and Theo., 42, 475205, 2009.

[37] P. Winternitz, Symmetry preserving discretization of differential equations and Lie point symmetries of differentialdifference equations, in D. Levi, P. J. Olver, Z. Thomova and P. Winternitz (editors) Symmetries and Integrability of Difference Equations, Cambridge University Press, Cambridge, pp. 292-341, 2011. 